\title{
Room-temperature sensing performance of hydrogen using palladium-based film by optical setup
}

\author{
YiYUN YAO ${ }^{1}$, Alain J. CORSO ${ }^{2,3}$, MARCO BAZZAN ${ }^{4}$, EnRICO TESSAROLO ${ }^{2}$, \\ ZhANSHAN WANG ${ }^{1}$, QIUSHI HUANG ${ }^{1}$, RUNZE QI $^{1}$, A. MARTUCCI ${ }^{5}$, MARIA G. PELIZZO ${ }^{2,3}$ \\ ${ }^{1}$ Key Laboratory of Advanced Micro Structural Materials MOE, \\ Institute of Precision Optical Engineering, School of Physics Science and Engineering, \\ Tongji University, 200092, Shanghai, China \\ ${ }^{2}$ National Research Council of Italy, Institute for Photonics and Nanotechnologies, \\ Via Trasea 7, 35131 Padova, Italy \\ ${ }^{3}$ University of Padova, Department of Information Engineering, \\ Via Gradenigo 6/B, 35131 Padova, Italy \\ ${ }^{4}$ University of Padova, Department of Physics and Astronomy, \\ Via Marzolo 19, 35131 Padova, Italy \\ ${ }^{5}$ University of Padova, Department of Industrial Engineering, \\ Via Gradenigo 6/A, 35131 Padova, Italy
}

Corresponding authors: pelizzo@dei.unipd.it (M.G. Pelizzo), alain.corso@pd.ifn.cnr.it (A.J. Corso)

\begin{abstract}
$\mathrm{H}_{2}$ sensing performance of novel Pd-Pt alloy films has been compared with those obtained by using $\mathrm{Pd}$ films and $\mathrm{H}_{2}$-reducted $\mathrm{PdO}$ films. Two different detecting systems were used to measure the hydrogenation and de-hydrogenation phases with a $\mathrm{H}_{2}$ concentration of both $5 \% \mathrm{v} / \mathrm{v}$ nitrogen and $1 \% \mathrm{v} / \mathrm{v}$ nitrogen at room temperature. The sensitivity loss observed for the $\mathrm{Pd}-\mathrm{Pt}$ alloy and $\mathrm{H}_{2}$-reducted PdO samples with respect to pure Pd samples can be explained in terms of the reduction in the lattice constant and interstitial volume due to the Pt addition, which determine a decrement of hydrogen atoms penetrating in the films. On the other hand, results show an improvement in time -response for $\mathrm{Pd}-\mathrm{Pt}$ alloy and $\mathrm{H}_{2}$-reducted $\mathrm{PdO}$ films with respect to pure Pd ones, presumably due to the increase of its permeability to $\mathrm{H}_{2}$. Moreover, the sensing measurements repeated after 60 days show that the Pd-Pt alloy films, unlike the Pd-based ones, fully preserve their performances, demonstrating the advantage of the Pt inclusion for stability purposes when the samples are stored upon humidity.
\end{abstract}

Keywords: hydrogen detection, palladium-based film, palladium-platinum alloy film, optical sensor.

\section{Introduction}

Hydrogen $\left(\mathrm{H}_{2}\right)$ has received a great deal of attention in numerous applications, including those which are interesting for chemical industry, clean fuels production and food pro- 
cessing $[\underline{1}-\underline{6}] . \mathrm{H}_{2}$ is a tasteless, odorless, colorless and inflammable gas which becomes explosive at concentrations higher than $4 \%$ in air, making the development of hydrogen sensors very crucial. There are several hydrogen sensing techniques, such as those based on thermal, electrochemical, mechanical, optical and acoustic effects; among them, the optical one is the most suitable and safe to be applied in the hydrogen atmosphere [ $[\underline{7}, \underline{8}]$. Palladium (Pd) is a widely used catalyst in the field of optical and conductive sensors since it demonstrates a valuable native sensitivity towards hydrogen. In fact, a significant change in the optical and electrical properties can be detected when Pd is exposed to hydrogen because of the formation of hydrides [-14]. The change in Pd optical response is due to absorption of atomic hydrogen, after decomposition of its molecules at the metal surface, and to the hydrogen atoms diffusion in the metal lattice [15-17]. In case of low partial pressure, the number of atoms diffused into $\mathrm{Pd}$ is moderate, the distance between the hydrogen atoms is large and the compositional strain induced in the Pd lattice is weak; such solid solution is called $\alpha$-phase. When the hydrogen partial pressure increases, the number of hydrogen atoms in the metal, as well as their mutual interactions resulting from lattice strain and electronic interactions, also increase, leading to the formation of the hydride, called $\beta$-phase. For hydrogen concentrations above $4 \%$, the hydride formation is considered complete at the expense of the $\alpha$-phase regions.

To improve the efficiency of the Pd sensors, different strategies were proposed, as well as various solutions to avoid temperature, humidity, and oxygen concentration performance dependence. Benefits in performance derive from higher film porosity and/or surface nano-structuring, which results in the increase of the effective surface-to-volume ratio [18-21]. However, Pd-based sensors stability is affected by the structural changes induced by the volume expansion [17]. Moreover, the response times of Pd-based sensors are determined by the dynamic equilibrium process involving hydrogen adsorption/desorption at the films surface, bulk diffusion and Pd hydride formation, which in case of pure Pd films results in limited performance.

The use of $\mathrm{Pd}$ alloy thin films such as $\mathrm{Pd}-\mathrm{Ag}, \mathrm{Pd}-\mathrm{Ni}$ and $\mathrm{Pd}-\mathrm{Y}$ has been demonstrated to reduce response and recovery times, due to the increased permeability of the alloy to $\mathrm{H}_{2}$, and to stabilize the properties of the sensor over time [2-26]. Platinum $(\mathrm{Pt})$ is also a common material applied in hydrogen sensing [27-30]. While Pd has good selectivity toward hydrogen but with poor stability, Pt has good stability in air and it can be used also as a protective layer of the sensing media [31, 32]. Nano-porous Pt samples have been also proven to have higher sensitivity with respect to pure platinum samples [ $\underline{33}$ ]. Given these premises, it is expected that Pd-Pt composite films can exhibit interesting catalyst properties and higher stability $[\underline{34}, \underline{35}]$. In the present work, we compare the sensing performance of novel $\mathrm{Pd}-\mathrm{Pt}$ alloy films with pure Pd films as well as those obtained by $\mathrm{H}_{2}$-reduction of $\mathrm{PdO}$, previously studied [20]. The Pd-Pt alloys operated reversibly upon $\mathrm{H}_{2}$ absorption and desorption. The absorbance change was characterized for two different $\mathrm{H}_{2}$ concentrations ( $1 \%$ or $5 \% \mathrm{v} / \mathrm{v}$ nitrogen) at room temperature. Two different detecting systems were used to record the hydrogenation and de-hydrogenation phases signal. The sensitivity loss with respect to pure Pd samples can be explained in terms of the reduction in the lattice constant and interstitial volume due to 
the Pt addition, which determine a decrement of hydrogen atoms penetrating in the alloy [22]. Interestingly, results show an improvement in time-response of Pd-Pt alloy films with respect to $\mathrm{Pd}$ ones, presumably due to the increase of its permeability to $\mathrm{H}_{2}$. Moreover, the sensing measurements repeated after 60 days show that the Pd-Pt alloy films, unlike the Pd-based ones, fully preserve their performances, demonstrating the advantage of the Pt inclusion for stability purposes.

\section{Materials and methods}

Palladium films were deposited on quartz substrate using e-beam evaporation technology. The base vacuum during the deposition process was about $10^{-4} \mathrm{~Pa}\left(10^{-6} \mathrm{mbar}\right)$ while the temperature of the substrate was controlled to be around $90^{\circ}$. The growth rate of the films was constant, being $0.5 \mathrm{~nm} / \mathrm{s}$. The final thickness of the Pd films was $12.7 \mathrm{~nm}$. Some of the samples were then oxidized by annealing at $600^{\circ}$ in a vacuum of about $1.5 \mathrm{mbar}$ for $2 \mathrm{~h}$; the annealing time was chosen in order to ensure the full oxidation of the film [르] . Afterwards, the oxidized samples were exposed for $15 \mathrm{~min}$ to $\mathrm{H}_{2}$ atmosphere ( $5 \% \mathrm{v} / \mathrm{v}$ nitrogen) at room temperature to obtain the reduced-Pd (r-Pd) samples by inducing the $\mathrm{H}_{2}$-reduction process described by the following chemical reaction:

$$
\mathrm{PdO}+\mathrm{H}_{2} \rightarrow \mathrm{Pd}+\mathrm{H}_{2} \mathrm{O}
$$

The Pd/Pt alloy (Pd-Pt_al) films were fabricated starting from Pd/Pt multilayer (Pt-Pt_ml) films [36]. Such multilayers were deposited on quartz substrates using direct current (DC) magnetron sputtering technology. The base vacuum was $2.5 \times 10^{-4} \mathrm{~Pa}$ $\left(2.5 \times 10^{-6} \mathrm{mbar}\right)$ and high purity argon $(99.999 \%)$ was used as working gas at a pressure of $0.200 \mathrm{~Pa}$. The effective deposition rates were $0.105 \mathrm{~nm} / \mathrm{s}$ for Pd and $0.145 \mathrm{~nm} / \mathrm{s}$ for Pt. The final structure of the multilayer was a stack of three $\operatorname{Pd}(2 \mathrm{~nm}) / \operatorname{Pt}(2 \mathrm{~nm})$ bi-layers over-coated with an additional Pd layer of $2 \mathrm{~nm}$. Afterwards, the mixture of the individual $\mathrm{Pd}$ and $\mathrm{Pt}$ layer was induced by means of two thermal and $\mathrm{H}_{2}$-reduction cycles. During the thermal process, the samples were annealed at $600^{\circ}$ for 2 hours in vacuum ( $1.5 \mathrm{mbar}$ ). During the $\mathrm{H}_{2}$-reduction process, the samples were exposed for 15 min to $\mathrm{H}_{2}$ atmosphere ( $5 \% \mathrm{v} / \mathrm{v}$ nitrogen) at room temperature.

After deposition, the thickness of the films was determined using a profilometer, KLA Tencor P-16+, and the morphology of the surface was evaluated by a non-contact Park-System XE-70 atomic force microscope (AFM). Furthermore, the Pd/Pt samples were characterized before and after each thermal/ $\mathrm{H}_{2}$-reduction process with $\mathrm{X}$-ray reflectivity (XRR) at the $\mathrm{Cu} \mathrm{K}-\alpha(\lambda=1.54 \AA)$ emission in order to confirm the formation of the Pd-Pt alloy. XRR were performed using a Philips MRD diffractometer, equipped with a parabolic multilayer mirror for primary beam conditioning. The reflected beam was measured as a function of the incidence angle by a proportional Xe counter, coupled to a parallel plate collimator with an angular acceptance of $0.01^{\circ}$. XRR measurements were taken for grazing angles ranging from $0.01^{\circ}$ to $3^{\circ}$, with a step of $0.005^{\circ}$. XRR curves were fitted using IMD software [ $\underline{37}$ ] assuming as free parameters the layer 


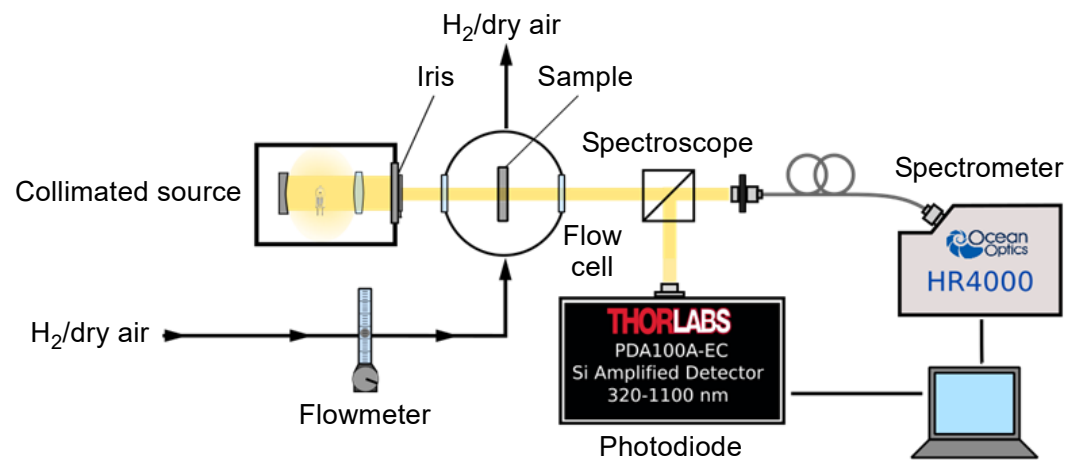

Fig. 1. Sketch of the setup to measure the gas sensing performance.

thicknesses, the material densities (both film and substrate), and the interface roughness. To carry out such fits, the Pd and Pt layers were modelled considering the atomic scattering factors provided by HENKE et al. [표]; the interface roughness was described by the Nevot-Croce model [37].

The optical transmittance of the films was measured using a double grating spectrophotometer (Varian Cary5000) in the 300-1700 nm spectral range.

The gas sensing performances of the as-deposited Pd, r-Pd and Pd-Pt_al films were tested in the 400-800 nm spectral range. A sketch of the experimental setup adopted is reported in Fig. 1. A sealed flow cell (with a volume of about $43 \mathrm{ml}$ ) is coupled with a flowmeter for monitoring the gas flux. The beam coming from a collimated halogen lamp is used as a test beam for the transmittance/absorbance measurements. The optical absorbance was measured by using an Ocean Optics USB4000 spectrometer and a Thorlabs PDA100A-EC photodiode simultaneously.

The tests were carried out by alternating the $\mathrm{H}_{2}(1 \%$ or $5 \% \mathrm{v} / \mathrm{v}$ nitrogen $)$ atmosphere with the dry air at room temperature $\left(22 \pm 2^{\circ}\right)$. The exposures sequences started by fluxing dry air into the flow cell for $5 \mathrm{~min}$ in order to set the initial atmosphere. Afterward, the $\mathrm{H}_{2}$ at the desired concentration was fluxed for $5 \mathrm{~min}$ and then replaced with dry air for another $5 \mathrm{~min}$. These two steps were then repeated many times. The flow rate was set as $0.8 \mathrm{l} / \mathrm{min}$ to ensure sufficient time and turbulence of gas recycling inside the flow cell and reacting with the sample.

The transmittance signal was acquired by a photodiode. The direct and transmitted beam signals were measured and the dynamic integrated absorbance $A_{\text {phot }}$ obtained. Addionally, for comparison, the dynamic relative integrated absorbance $A_{\Delta \lambda}$ was obtained by making the integral over the 400-800 $\mathrm{nm}$ range of the absorbance values $A(\lambda)=\log \left(I_{0}(\lambda)\right) /\left(I_{\mathrm{T}}(\lambda)\right)$, where $I_{\mathrm{T}}(\lambda)$ is the transmittance signal provided by the spectrometer and $I_{0}(\lambda)$ the reference one:

$$
A_{\Delta \lambda}=\frac{1}{\lambda_{2}-\lambda_{1}} \int_{\lambda_{1}}^{\lambda_{2}} A(\lambda) \mathrm{d} \lambda
$$




\section{Results and discussions}

The thickness of the Pd films measured prior, after annealing and after $\mathrm{H}_{2}$-reduction is reported in the Table. Since Pd crystallizes in a $f c c$ structure $(a=3.882 \AA$ with four atoms per cubic unit cell) and $\mathrm{PdO}$ has a tetragonal structure $(a=3.043 \AA, c=5.336 \AA$ with two Pd atoms per unit cell), the thickness of a Pd film that is completely oxidized to $\mathrm{PdO}$ should increase by a factor of $\left(4\left(a^{2} c\right)_{\mathrm{PdO}}\right) / 2\left(a^{3}\right)_{\mathrm{Pd}}=1.68[\underline{20}]$. However, the annealed films considered in the present study increased by a factor ranging from 3.7-4.2 with respect to the original thickness, suggesting that the $\mathrm{PdO}$ film is highly porous. This porosity is preserved also after the $\mathrm{H}_{2}$-reduction, as the contraction undergoing by the films does not restore the original thickness.

$\mathrm{T}$ a b 1 e. Pd and $\mathrm{Pd} / \mathrm{Pt}$ typical sample thickness along the annealing $/ \mathrm{H}_{2}$-reduction process.

\begin{tabular}{lll}
\hline & \multicolumn{2}{c}{ Thickness [nm] } \\
\cline { 2 - 3 } Process & Pd sample & Pd/Pt sample \\
\hline As deposited & 12.7 & 14.4 \\
After oxidation & 51.0 & 24.7 \\
After reduction & 38.3 & 18.3 \\
\hline
\end{tabular}

The Pd/Pt samples thickness changes along the processes is reported in the Table. Pt crystalizes in a $f c c$ structure (volume $=a^{3}, a=3.92 \AA$ and four atoms per unit cell) and its oxide is formed at temperatures higher than $870^{\circ} \mathrm{C}$. It is thus reasonable to consider that the adopted thermal process cannot induce the Pt oxidation and the annealed films will be composed by an alloy of Pt and PdO. Based on this assumption, the thickness expansion which underwent after thermal process should be of a factor of

$$
\frac{\frac{1}{2}\left(a^{2} c\right)_{\mathrm{PdO}}+\left(\frac{N_{\mathrm{Pt}}}{N_{\mathrm{Pd}}}\right) \frac{1}{4}\left(a^{3}\right)_{\mathrm{Pt}}}{\frac{1}{4}\left(a^{3}\right)_{\mathrm{Pd}}+\left(\frac{N_{\mathrm{Pt}}}{N_{\mathrm{Pd}}}\right) \frac{1}{4}\left(a^{3}\right)_{\mathrm{Pt}}}=1.39
$$

where $N_{\mathrm{Pt}}$ and $N_{\mathrm{Pd}}$ are the atomic number density of Pt and Pd, respectively. However, all the films considered in the present study showed a thickness increase of a factor ranging from 1.71 to 1.77 , slightly higher than the theoretical one. Again, this discrepancy can be still explained considering an increase of the porosity due to the thermal process.

The XRR curves of a Pd/Pt sample before and after annealing/ $\mathrm{H}_{2}$-reduction cycles are reported in Fig. 2. In the as-deposited Pd/Pt sample, the XRR was fitted by considering a multilayer model with a period thickness of $4.02 \mathrm{~nm}$, a thickness ratio $d_{\mathrm{Pd}} /\left(d_{\mathrm{Pd}}+d_{\mathrm{Pt}}\right)$ of 0.50 , a Pd density $\rho_{\mathrm{Pd}}$ of $11.95 \mathrm{~g} / \mathrm{cm}^{3}$, a Pt density $\rho_{\mathrm{Pt}}$ of $20.58 \mathrm{~g} / \mathrm{cm}^{3}$, a Pt on Pd roughness $\sigma_{1}$ of $0.20 \mathrm{~nm}$ and a Pd on Pt roughness $\sigma_{2}$ of $0.26 \mathrm{~nm}$. On the 

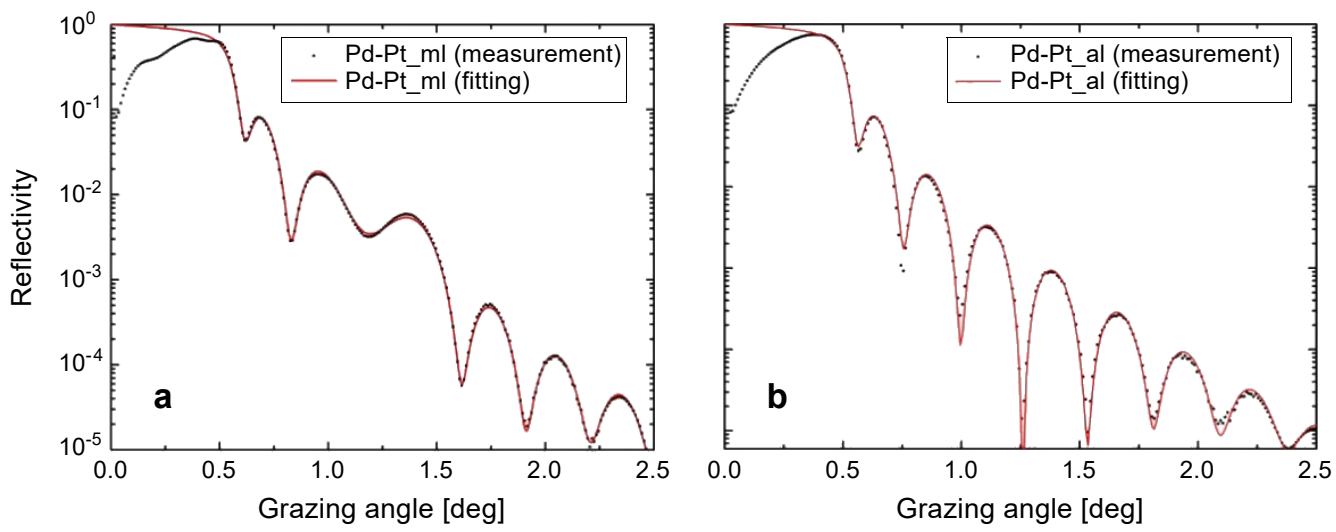

Fig. 2. XRR result of the Pd/Pt sample before and after processes. Before: Pd-Pt_ml film (a), after: Pd-Pt_al film (b).

other hand, the XRR measured after the thermal $/ \mathrm{H}_{2}$-reduction cycle revealed a curve which can be successfully fitted with a Pd-Pt alloy monolayer model with a thickness of $23.01 \mathrm{~nm}$ and a density of $13.43 \mathrm{~g} / \mathrm{cm}^{3}$. The retrieved film density, which is lower
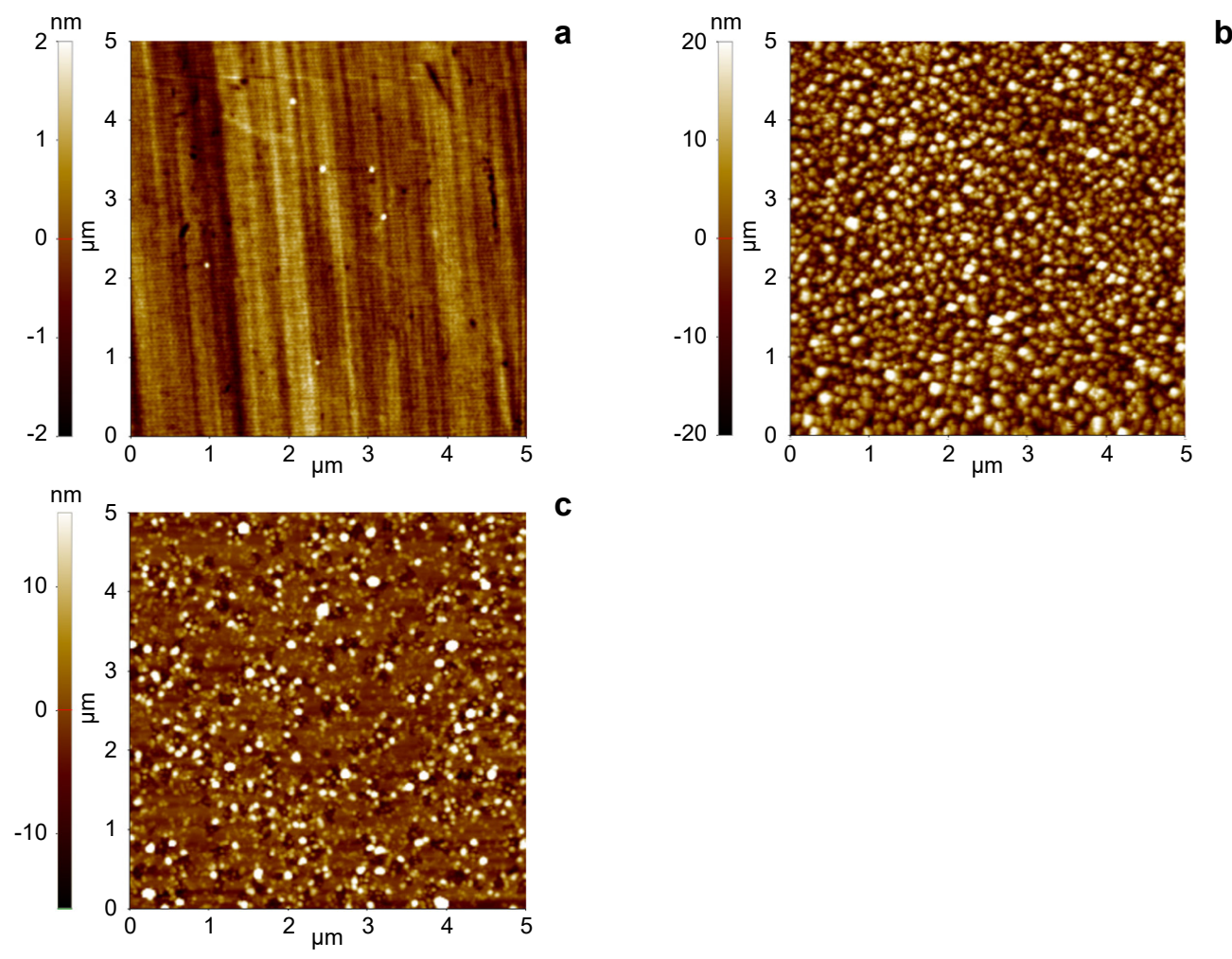

C

Fig. 3. The topography of all the samples by AFM. Pd film, roughness $r_{\mathrm{q}}=0.525 \mathrm{~nm}(\mathbf{a}), \mathrm{r}-\mathrm{Pd}$ film, roughness $r_{\mathrm{q}}=8.954 \mathrm{~nm}(\mathbf{b})$, and Pd-Pt_al film, roughness $r_{\mathrm{q}}=6.305 \mathrm{~nm}(\mathbf{c})$. 

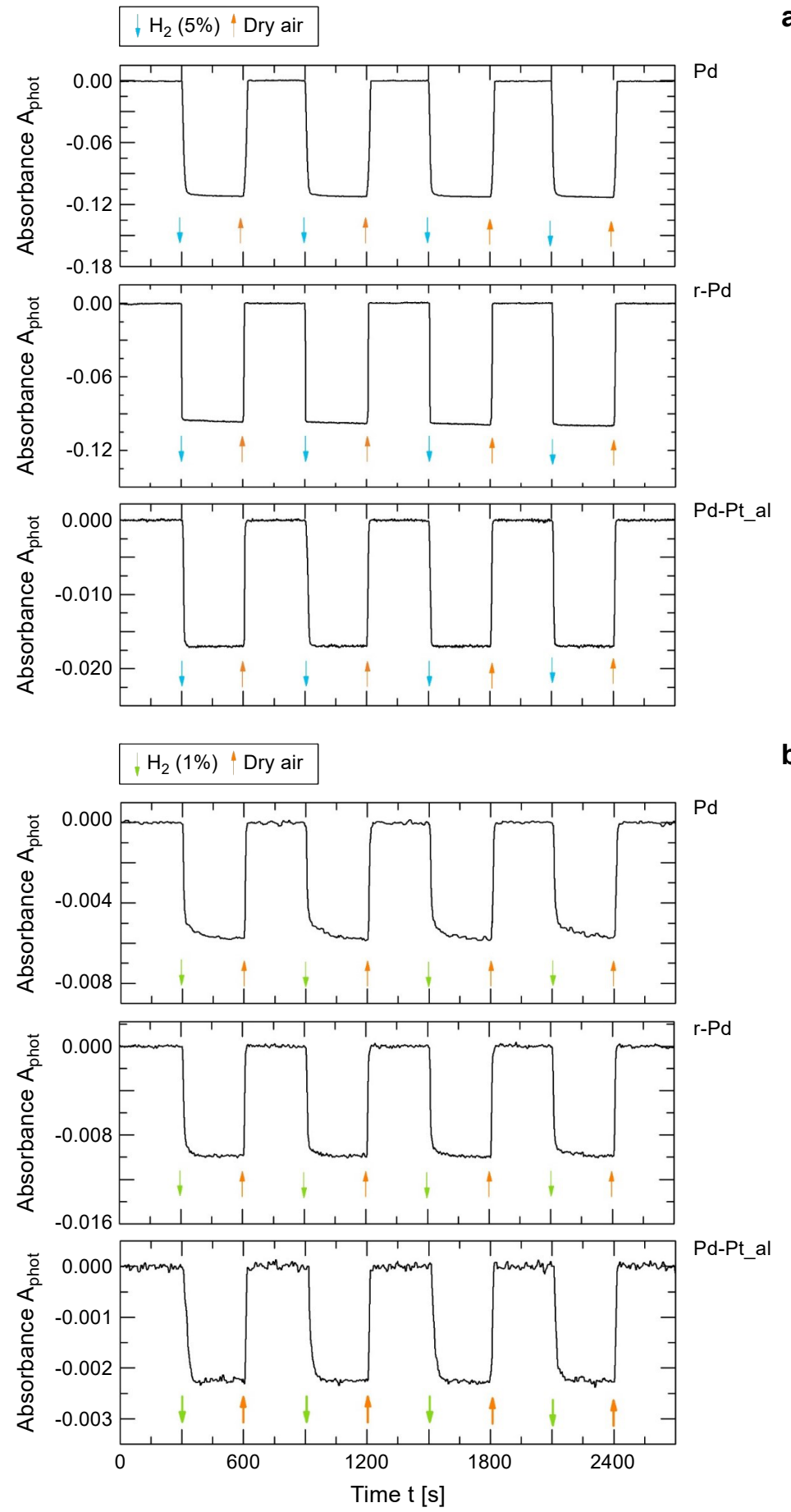

Fig. 4. The dynamic integrated absorbance $A_{\text {phot }}$ of Pd, r-Pd and Pd-Pt_al samples along different cycles. $\mathrm{H}_{2}$ concentration of $5 \%(\mathbf{a})$ and of $1 \%(\mathbf{b})$. 
with respect to the theoretical one (i.e. $\rho=15.64 \mathrm{~g} / \mathrm{cm}^{3}$ ), and the final thickness, which is higher than the initial value, suggests that the films are still porous.

The morphologies of all the samples are investigated by AFM as showed in Fig. 3. Nano-bubbles appear on the surface of Pd-Pt_al and r-Pd samples, as confirmed by the root mean square (RMS) roughness data. The lines of the as-deposited Pd sample come from the fabrication of the substrate.

The dynamic integrated absorbance $A_{\text {phot }}$ of the Pd, r-Pd and Pd-Pt_al was acquired along different cycles. The results are reported in Fig. 4 a for the case $5 \% \mathrm{H}_{2}$ concentration and Fig. $4 \mathbf{b}$ for the case $1 \% \mathrm{H}_{2}$ concentration.

According to the profiles in Fig. 4, three different features have been analyzed: the OAC (optical absorbance change), calculated as the average of the steps for each cycle; the response time, calculated as the average time to the change from $5 \%$ to $95 \%$ of the absorbance when switching form air to $\mathrm{H}_{2}$; the recovery time, calculated as the average time to the change from $5 \%$ to $95 \%$ of the absorbance when switching from $\mathrm{H}_{2}$ to air.

The OAC, representing the sensitivity of the sample, is reported in Fig. 5. As expected, the Pd-Pt_al sample shows lower sensitivity than both the Pd and r-Pd ones if we consider the $\overline{5 \%} \mathrm{H}_{2}$ concentration case; on the contrary, it shows comparable performance for the case of $1 \% \mathrm{H}_{2}$ concentration. In this last case, the r-Pd offers the better results. The sensitivity loss of Pd-Pt_al with respect to pure Pd and r-Pd samples can be explained in terms of the reduction in the lattice constant and interstitial volume due to the Pt addition.

The response time and recovery time for all the different samples (Pd, r-Pd and Pd-Pt_al) are plotted in Fig. 6. Interestingly, the response of the treated samples, means $\mathrm{Pd}-\mathrm{Pt}$ - al and r-Pd, are quicker than the Pd virgin sample, especially when considering the recovery time in the $5 \% \mathrm{H}_{2}$ concentration case. This can be explained in terms of induced porosity in the treated samples, which allows a higher gas flushing through the films. On the other hand, the reduction of interstitial volume due to the Pt addition in Pd-Pt_al sample decreases the performance of it with respect to the r-Pd sample.

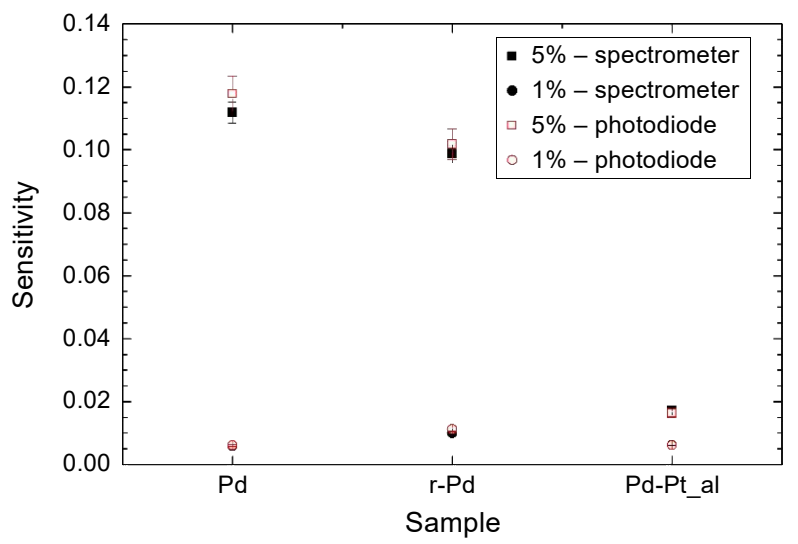

Fig. 5. Sensitivity of Pd, r-Pd and Pd-Pt_al samples along different cycles with a $\mathrm{H}_{2}$ concentration of 5\% and $1 \%$ measured both with spectrometer and photodiode. 


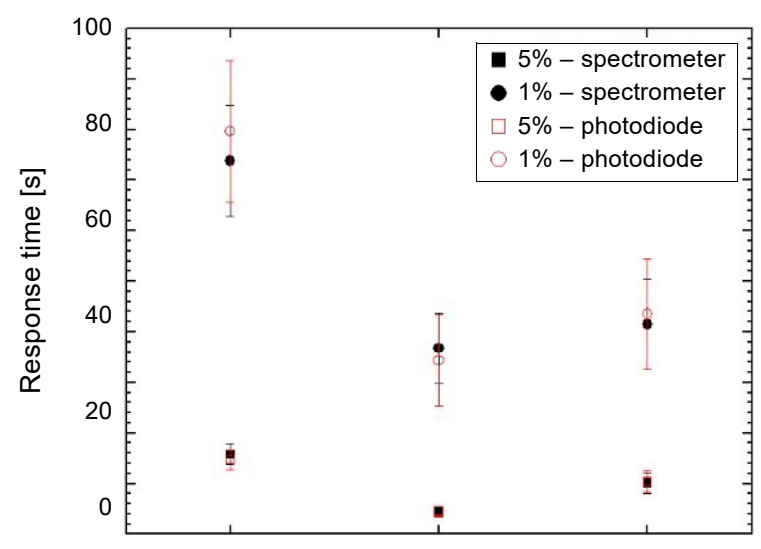

a

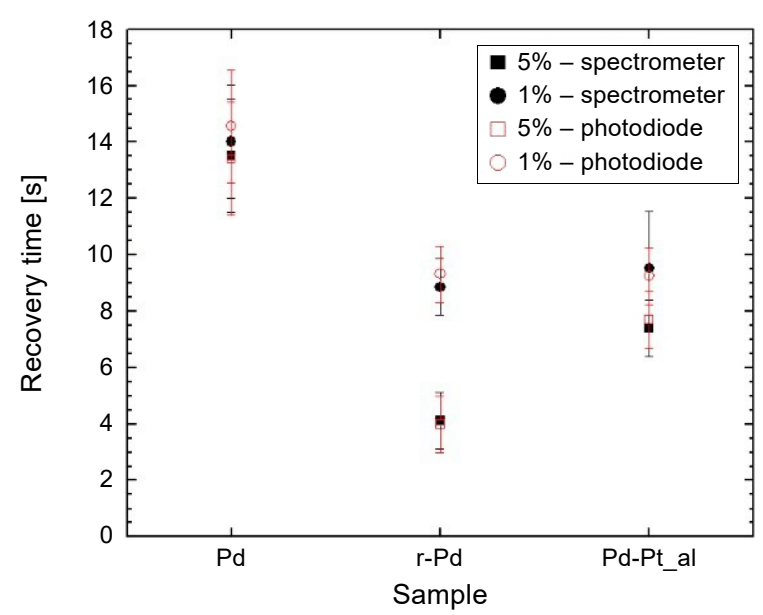

b

Fig. 6. Response performance of all samples with $\mathrm{H}_{2}$ concentration of $5 \%$ and $1 \%$. Response (a) and recovery (b) times.

Considering that the performance obtained from the r-Pd sensors are comparable or, even, better than standard Pd sensors, the aging effects induced by regular environment storing have been investigated by considering the r-Pd samples as representative of the pure Pd-based sensors. The Pd-Pt_al and r-Pd samples have been stored in air at ambient temperature for 60 days, and response tests then repeated (Fig. 7). In previous studies, it has been demonstrated that Pd-based hydrogen sensors are highly affected by the environmental humidity, reducing the sensibility and the response time [26, $\underline{39}]$. These effects are mainly due to the Pd surface-catalyzed reactions with $\mathrm{H}_{2} \mathrm{O}$ occurring when a Pd film with a thin layer of $\mathrm{H}_{2} \mathrm{O}$ on top reacts with $\mathrm{H}_{2}$ [26]. Similarly to what has been observed in previous studies for the standard Pd-based sensors, the reduction of performance due to humidity is clearly recognizable also in r-Pd samples tested after 60-days in regular environment (see Fig. 7). The use of Pt alloyed with Pd contributes to reduce the impact of the surface reactions on the sensing performance. In fact, the cycle of Pd-Pt_al sample is complete from the second cycle while that of 

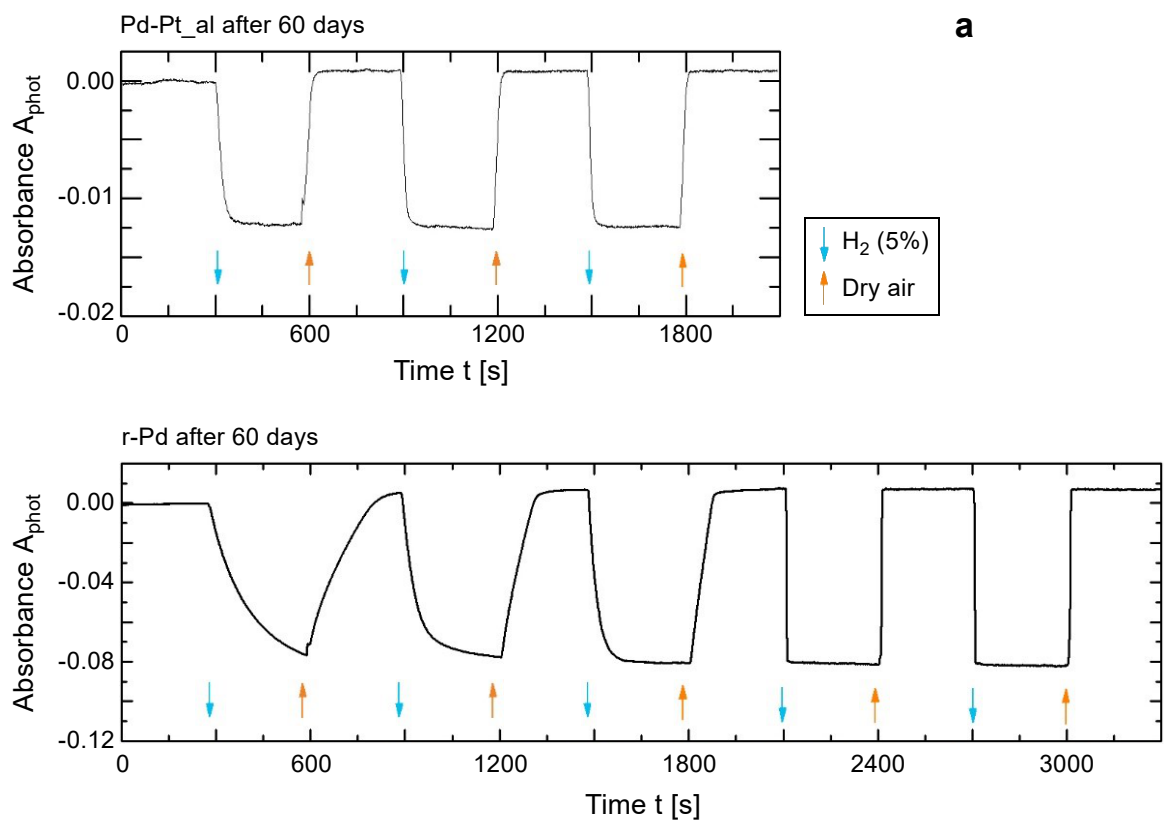

b

Fig. 7. The sensing performance cycles of samples Pd-Pt_al (Pd/Pt alloy film) and r-Pd (reduced Pd film) after 60 days.

r-Pd sample is complete from the fourth cycle, showing the improved stability obtained in the Pd-Pt_al.

\section{Conclusions}

The hydrogen sensing performance of Pd, r-Pd and Pt-Pd_al thin film samples has been investigated. The r-Pd samples have been fabricated by high-temperature reaction and reduction process starting from a palladium thin film; the Pd-Pt alloy monolayer films have been fabricated by inducing an intermixing of the layers of $\mathrm{Pd} / \mathrm{Pt}$ multilayers using two cycles of high-temperature reaction and reduction process. The samples have been tested by measuring the modification of the absorbance in alternative atmospheres of $\mathrm{H}_{2}(1 \%$ or $5 \% \mathrm{v} / \mathrm{v}$ nitrogen $)$ and dry air at room temperature $\left(22 \pm 2^{\circ} \mathrm{C}\right)$. The data have been acquired simultaneously by a spectrometer and a photodiode, which provide two independent sets of measurement with an excellent agreement. The response and recovery time of the treated sample are quicker than the standard palladium film one, due to the increase of the porosity of the films. The Pd-Pt_al film shows lower sensitivity than the Pd film r-Pd film due to the lower concentration of adsorption sites of hydrogen. The r-Pd shows enhanced sensitivity at $1 \%$ hydrogen for the increase of the effective surface-to-volume ratio. After 60 days of the sample exploring in air, the cycle of Pd-Pt_al sample completes quicker than the cycle of r-Pd sample, showing the stability profit from the doping of platinum. 


\section{References}

[1] Gupta R., Basile A., Veziroglu T.N., Compendium of Hydrogen Energy: Hydrogen Storage, Distribution and Infrastructure, Woodhead Publishing, 2016.

[2] Schlapbach L., Hydrogen-fuelled vehicles, Nature 460, 2009, pp. 809-811, DOI: 10.1038/460809a.

[3] Chu S., Majumdar A., Opportunities and challenges for a sustainable energy future, Nature 488, 2012, pp. 294-303, DOI: 10.1038/nature11475.

[4] Cecere D., Giacomazzi E., Ingenito A., A review on hydrogen industrial aerospace applications, International Journal of Hydrogen Energy 39(20), 2014, pp. 10731-10747, DOI: 10.1016/ j.ijhydene.2014.04.126.

[5] Ahluwalia R.K., Peng J.K., Roh H.S., Hua T.Q., Houchins C., James B.D., Supercritical cryo-compressed hydrogen storage for fuel cell electric buses, International Journal of Hydrogen Energy 43(22), 2018, pp. 10215-10231, DOI: 10.1016/j.ijhydene.2018.04.113.

[6] Cottrell C.A., Grasman S.E., Thomas M., Martin K.B., Sheffield J.W., Strategies for stationary and portable fuel cell markets, International Journal of Hydrogen Energy 36(13), 2011, pp. 7969 -7975, DOI: $\underline{10.1016 / j . i j h y d e n e .2011 .01 .056 . ~}$

[7] Hübert T., Boon-Brett L., Black G., Banach U., Hydrogen sensors - A review, Sensors and Actuators B: Chemical 157(2), 2011, pp. 329-352, DOI: 10.1016/j.snb.2011.04.070.

[8] Buttner W.J., Post M.B., Burgess R., Rivkin C., An overview of hydrogen safety sensors and requirements, International Journal of Hydrogen Energy 36(3), 2011, pp. 2462-2470, DOI: 10.1016/ j.ijhydene.2010.04.176.

[9] Wadell C., Syrenova S., Langhammer C., Plasmonic hydrogen sensing with nanostructured metal hydrides, ACS Nano 8(12), 2014, pp. 11925-11940, DOI: 10.1021/nn505804f.

[10] Tittl A., Mai P., Taubert R., Dregely D., Liu N., Giessen H., Palladium-based plasmonic perfect absorber in the visible wavelength range and its application to hydrogen sensing, Nano Letters 11(10), 2011, pp. 4366-4369, DOI: 10.1021/n1202489g.

[11] Cerea A., Garoli D., Zilio P., Dipalo M., Calandrini E., Jacassi A., Caprettini V., Carrara A., Pelizzo M.G., De Angelis F., Modified three-dimensional nanoantennas for infrared hydrogen detection, Microelectronic Engineering 162, 2016, pp. 105-109, DOI: 10.1016/j.mee.2016.05.004.

[12] Yuan M., Lee K., Van Campen D.G., Liguori S., Toney M.F., Wilcox J., Hydrogen purification in palladium-based membranes: an operando X-ray diffraction study, Industrial \& Engineering Chemistry 58(2), 2019, pp. 926-934, DOI: 10.1021/acs.iecr.8b05017.

[13] Zuccon S., Zuppella P., Cristofani M., Silvestrini S., Corso A.J., Maggini M., Pelizzo M.G., Functional palladium metal films for plasmonic devices: an experimental proof, Journal of Optics 16(5), 2014, article 055001, DOI: 10.1088/2040-8978/16/5/055001.

[14] ÖZtürk S., KILInç N., Pd thin films on flexible substrate for hydrogen sensor, Journal of Alloys and Compounds 674, 2016, pp. 179-184, DOI: 10.1016/j.jallcom.2016.03.042.

[15] Li Y., CHENG Y.T., Hydrogen diffusion and solubility in palladium thin films, International Journal of Hydrogen Energy 21(4), 1996, pp. 281-291, DOI: 10.1016/0360-3199(95)00094-1.

[16] LeE E., LeE J.M., Koo J., LEE W., LeE T., Hysteresis behavior of electrical resistance in Pd thin films during the process of absorption and desorption of hydrogen gas, International Journal of Hydrogen Energy 35(13), 2010, pp. 6984-6991, DOI: 10.1016/j.ijhydene.2010.04.051.

[17] Corso A.J., Martucci A., Bazzan M., Zuppella P., Garoli D., Pelizzo M.G., In situ real-time investigation of hydrogen-induced structural and optical changes in palladium thin films, Journal of Alloys and Compounds 704, 2017, pp. 303-310, DOI: 10.1016/j.jallcom.2017.02.042.

[18] LeE Y.T., LeE J.M., Kim Y.J., Joe J.H., LeE W., Hydrogen gas sensing properties of PdO thin films with nano-sized cracks, Nanotechnology 21(16), 2010, article 165503, DOI: $10.1088 / 0957-4484 / 21 /$ $\underline{16 / 165503 .}$.

[19] Noh H.J., Kim H.J., Park Y.M., Park J.S., Lee H.N., Complex behavior of hydrogen sensor using nanoporous palladium film prepared by evaporation, Applied Surface Science 480, 2019, pp. 52-56, DOI: 10.1016/j.apsusc.2019.02.088. 
[20] Corso A.J., Tessarolo E., Guidolin M., Della Gaspera E., Martucci A., Angiola M., Donazzan A., Pelizzo M.G., Room-temperature optical detection of hydrogen gas using palladium nano-islands, International Journal of Hydrogen Energy 43(11), 2018, pp. 5783-5792, DOI: 10.1016/j.ijhydene. 2018.01.183.

[21] Boudiba A., Zhang C., Umek P., Bittencourt C., Snyders R., Olivier M.G., Debliquy M., Sensitive and rapid hydrogen sensors based on $\mathrm{Pd}-\mathrm{WO}_{3}$ thick films with different morphologies, International Journal of Hydrogen Energy 38(5), 2013, pp. 2565-2577, DOI: 10.1016/j.ijhydene.2012.11.040.

[22] Lee E., Lee J.M., Lee E., Noh J.S., Joe J.H., Jung B., LeE W., Hydrogen gas sensing performance of $P d-N i$ alloy thin films, Thin Solid Films 519(2), 2010, pp. 880-884, DOI: 10.1016/j.tsf.2010.07.122.

[23] Liu Y., Chen Y., Song H., Zhang G., Hydrogen gas sensor based on palladium and yttrium alloy ultrathin film, Review of Scientific Instruments 83(12), 2012, article 125003, DOI: 10.1063/1.4770329.

[24] Sharma B., Kim J.S., Pd/Ag alloy as an application for hydrogen sensing, International Journal of Hydrogen Energy 42(40), 2017, pp. 25446-25452, DOI: 10.1016/j.ijhydene.2017.08.142.

[25] Nugroho F.A.A., Darmadi I., Zhdanov V.P., Langhammer C., Universal scaling and design rules of hydrogen-induced optical properties in Pd and Pd-alloy nanoparticles, ACS Nano 12(10), 2018, pp. 9903-9912, DOI: 10.1021/acsnano.8b02835.

[26] Zhao Z., Knight M., Kumar S., Eisenbraun E.T., Carpenter M.A., Humidity effects on Pd/Au-based all-optical hydrogen sensors, Sensors and Actuators B: Chemical 129(2), 2008, pp. 726-733, DOI: 10.1016/j.snb.2007.09.032.

[27] Dai J., Peng W., Wang G., Xiang F., Qin Y., Wang M., Dai Y., Yang M., Deng H., Zhang P., Ultra -high sensitive optical fiber hydrogen sensor using self-referenced demodulation method and $\mathrm{WO}_{3}$ -Pd ${ }_{2}$ Pt-Pt composite film, Optics Express 25(3), 2017, pp. 2009-2015, DOI: 10.1364/OE.25.002009.

[28] Cao F., Zhao P., Wang Z., Zhang X., Zheng H., Wang J., Zhou D., Hu Y., Gu H., An ultrasensitive and ultraselective hydrogen sensor based on defect-dominated electron scattering in Pt nanowire arrays, Advanced Materials Interfaces 6(1), 2018, article 1801304, DOI: 10.1002/admi.201801304.

[29] Sripada R., Parambath V.B., Baro M., Nair S.P.N., Sundara R., Platinum and platinum-iron alloy nanoparticles dispersed nitrogen-doped graphene as high performance room temperature hydrogen sensor, International Journal of Hydrogen Energy 40(32), 2015, pp. 10346-10353, DOI: 10.1016/ j.ijhydene.2015.06.018.

[30] Yang M., Qin Y., Ma Y., Wang G., Xiang F., Wang M., Dai J., Chen Z., Xia J., Zhou L., High-sensitivity fiber optic hydrogen sensor in air by optimizing a self-referenced demodulating method, Applied Optics 57(27), 2018, pp. 8011-8015, DOI: 10.1364/AO.57.008011.

[31] Dai J., Yang M., Yang Z., Li Z., Wang Y., Wang G., Zhang Y., Zhuang Z., Performance of fiber Bragg grating hydrogen sensor coated with Pt-loaded $\mathrm{WO}_{3}$ coating, Sensors and Actuators B: Chemical 190, 2014, pp. 657-663, DOI: 10.1016/j.snb.2013.08.083.

[32] Strohfeldt N., Tittl A., Giessen H., Long-term stability of capped and buffered palladium-nickel thin films and nanostructures for plasmonic hydrogen sensing applications, Optical Materials Express 3(2), 2013, pp. 194-204, DOI: 10.1364/OME.3.000194.

[33] Abburi A., Abrams N., Yeh W.J., Synthesis of nanoporous platinum thin films and application as hydrogen sensor, Journal of Porous Materials 19(5), 2012, pp. 543-549, DOI: 10.1007/s10934-011 -9503-8.

[34] Shim J.Y., Lee J.D., Jin J.M., CheOng H., LeE S.H., Pd-Pt alloy as a catalyst in gasochromic thin films for hydrogen sensors, Solar Energy Materials and Solar Cells 93(12), 2009, pp. 2133-2137, DOI: $10.1016 /$ j.solmat.2009.01.004.

[35] Lebon A., García-Fuente A., Vega A., Aguilera-Granja F., Hydrogen interaction in Pd-Pt alloy nanoparticles, The Journal of Physical Chemistry C 116(1), 2012, pp. 126-133, DOI: 10.1021/ ip207329q.

[36] Gautam Y.K., Sanger A., Kumar A., Chandra R., A room temperature hydrogen sensor based on $P d-M g$ alloy and multilayers prepared by magnetron sputtering, International Journal of Hydrogen Energy 40(45), 2015, pp. 15549-15555, DOI: 10.1016/j.ijhydene.2015.08.078. 
[37] Windt D.L., IMD —Software for modeling the optical properties of multilayer films, Computers in Physics 12(4), 1998, pp. 360-370, DOI: 10.1063/1.168689.

[38] Henke B.L., Gullikson E.M., Davis J.C., X-ray interactions: photoabsorption, scattering, transmission and reflection at $E=50-30,000 \mathrm{eV}, Z=1-92$, Atomic Data and Nuclear Data Tables 54(2), 1993, pp. 181-342, DOI: 10.1006/adnd.1993.1013.

[39] Hughes R.C., Schubert W.K., Buss R.J., Solid-state hydrogen sensors using palladium-nickel alloys: effect of alloy composition on sensor response, Journal of the Electrochemical Society 142(1), 1995, pp. 249-254, DOI: 10.1149/1.2043887.

Received October 30, 2019 in revised form January 21, 2020 